\title{
Primary sarcomatoid carcinoma of the mandibular gingiva: clinicopathological and radiological findings
}

Xun-ze Shen ${ }^{1}$, MD, Fang Liü $^{2}$, MD

\begin{abstract}
Sarcomatoid carcinoma is a rare, malignant biphasic neoplasm with an epithelial and a spindle cell component. Primary sarcomatoid carcinomas arising from mandibular gingiva are known to be extremely rare, with only one case reported to date. Herein, we discuss the radiographic and computed tomographic appearances and pathological features of primary mandibular sarcomatoid carcinoma, which was confirmed by clinicopathology, in a 72-year-old man. In addition, we present a brief review of the relevant literature.
\end{abstract}

Keywords: computed tomography, mandible gingiva, primary carcinoma, radiography, sarcomatoid carcinoma

\section{INTRODUCTION}

Sarcomatoid carcinoma is a rare biphasic tumour with both malignant epithelial (carcinomatous) and mesenchymal (sarcomatous) components. It usually occurs in the urinary tract and upper respiratory tract in elderly men, and is rarely found in the maxillofacial region. ${ }^{(1,2)}$ Primary sarcomatoid carcinoma of the mandibular gingiva is extremely rare. To the best of our knowledge, only one such case has been clinically reported in the literature, either as 'sarcomatoid carcinoma' or 'carcinosarcoma'. ${ }^{(3)}$ Herein, we discuss the appearance of primary sarcomatoid carcinoma in the left mandible on radiography and computed tomography (CT), as well as the pathological features of the tumour.

\section{CASE REPORT}

A 72-year-old man presented with a hard, nonmobile, painless mass in the left mandibular region. The patient had discovered the mass incidentally two months earlier, but reported no paraesthesia or limitation of mouth opening and food intake at that time. Recently, he felt that the mass had grown significantly and caused a feeling of numbness and distension in the left lower lip. Physical examination showed missing jaw teeth and a $40 \mathrm{~mm} \times 50 \mathrm{~mm}$, poorly defined and indurated tumour without tenderness.

Panoramic radiography revealed diffuse bone loss in the left mandibular body without sclerotic margins. The focus range was about $40 \mathrm{~mm} \times 20 \mathrm{~mm}$, its edge uneven, and the cortical bone eroded and destroyed. In the right mandibular body, a $25 \mathrm{~mm} \times$ $15 \mathrm{~mm}$ gourd-shaped area of localised bone loss with sclerotic margins was seen, and the lesion had a smooth, well-defined border (Fig. 1).

Plain CT of the left mandibular body revealed a soft tissue mass, with attenuation that was slightly lower than that of the muscle, and bony destruction was also evident. There was no trabecular bone structure, and the boundary between the lesion and adjacent muscle was unclear. In the right mandibular body, plain CT revealed a loculated radiolucent lesion with complete
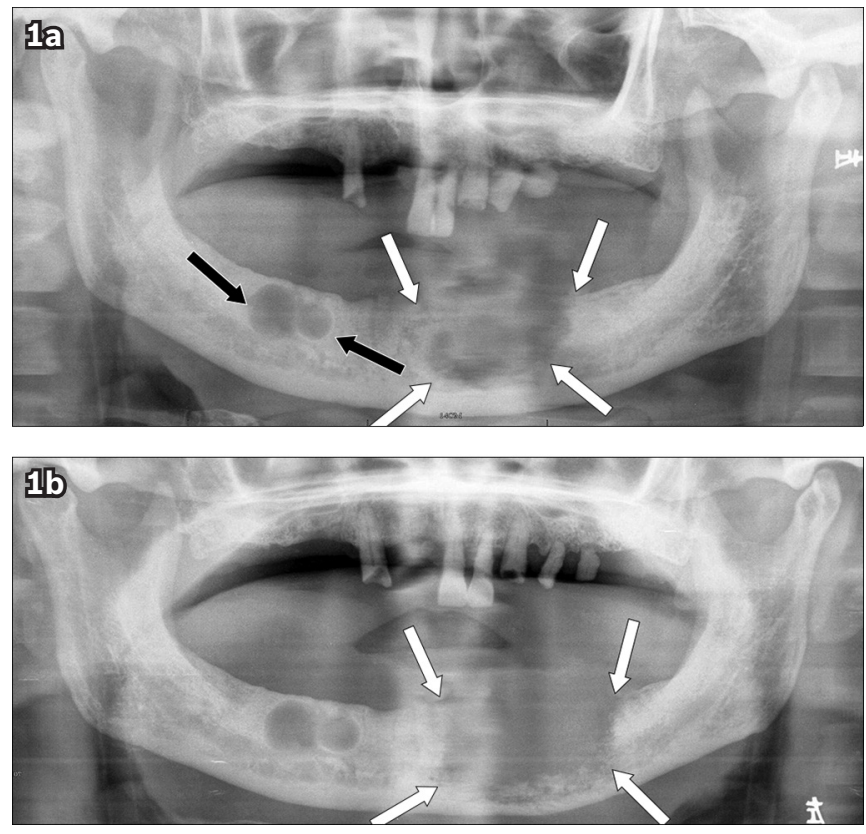

Fig. 1 Panoramic radiographs show (a) diffuse, irregular osteolytic bone destruction without sclerotic margins, marking the lesion of sarcomatoid carcinoma (white arrows) in the left mandibular body, and a gourd-shaped area of localised bone loss with sclerotic margins, marking the lesion of epithelial odontogenic cyst (black arrows) in the right mandibular body; and (b) a markedly enlarged lesion in the left mandibular body (white arrows) and an unchanged lesion in the right mandible upon re-examination one month later.

edges but without signs of expansionary growth (Fig. 2). Chest radiography did not reveal any abnormalities.

Subsequently, complete gross excision of the left mandibular tumour was carried out. The tumour showed expansionary growth, and complete erosion of the bone wall on the labial and lingual sides was observed. A non-encapsulated mass measuring $30 \mathrm{~mm} \times 30 \mathrm{~mm}$ in diameter with central necrosis was detected. The whole tumour mass was detached using a periosteal elevator along the bone wall. Histopathology of the excised tumour mass revealed an infiltrative and destructive growing neoplasm, characterised by malignant epithelial and spindle stromal elements. The epithelial element showed squamous

${ }^{1}$ Department of Radiology, ${ }^{2}$ Department of Pathology, Shaoxing People's Hospital, Zhejiang, China

Correspondence: Dr Shen Xun-ze, Associate Chief Physician, Department of Radiology, Shaoxing People's Hospital, No. 568, Zhongxing North Road, Shaoxing City, Zhejiang Province 312000, China. shenxunze@163.com 

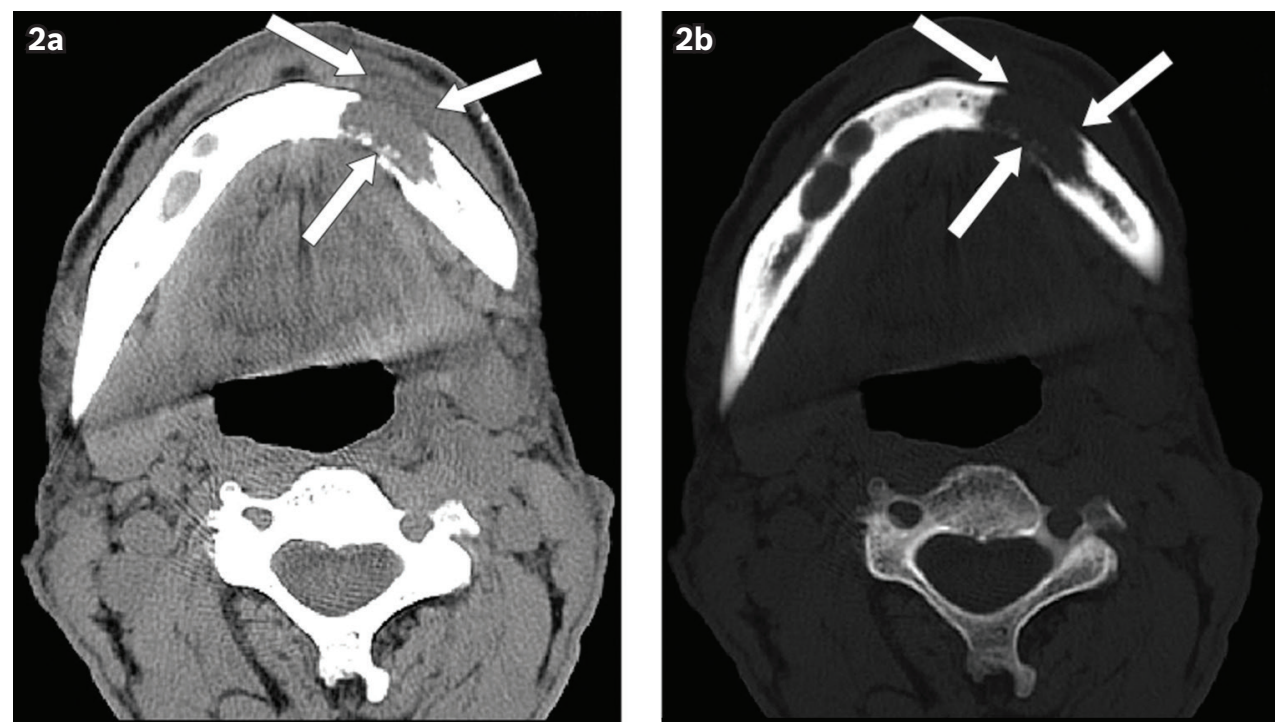

Fig. 2 Axial unenhanced CT images show (a) the dimension of the slightly lower density tumour mass (arrows) in the soft-tissue window; and (b) cortical bone erosion and destruction of the left horizontal ramus (arrows) in the bone window.
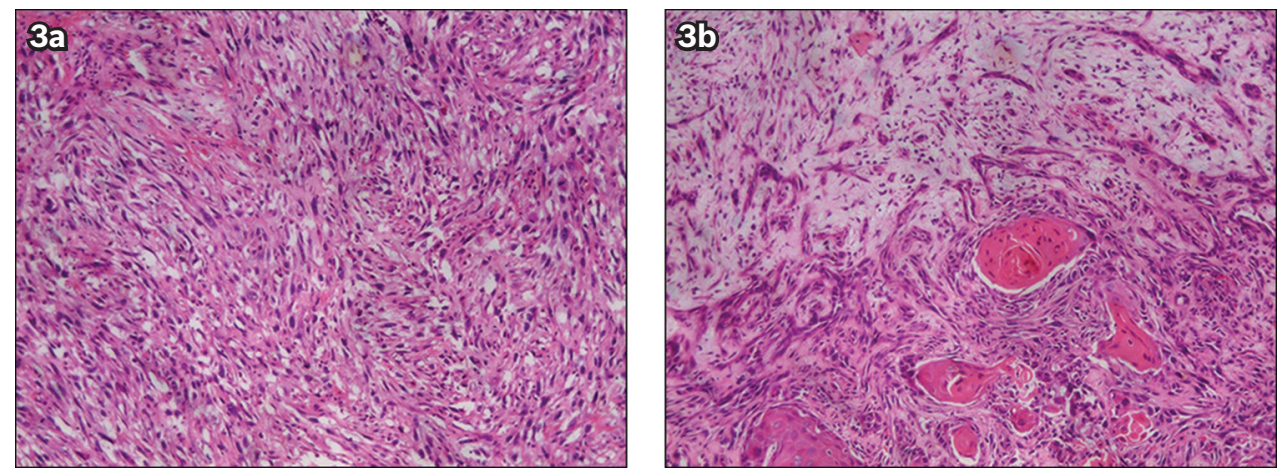

Fig. 3 Photomicrographs of the excised tumour mass show (a) a sarcomatous component with hypercellularity, marked pleomorphism and bizarre, enlarged nuclei; and (b) the transitional zone between the squamous carcinoma and sarcoma components (Haematoxylin \& eosin, $\times 200$ for both images).
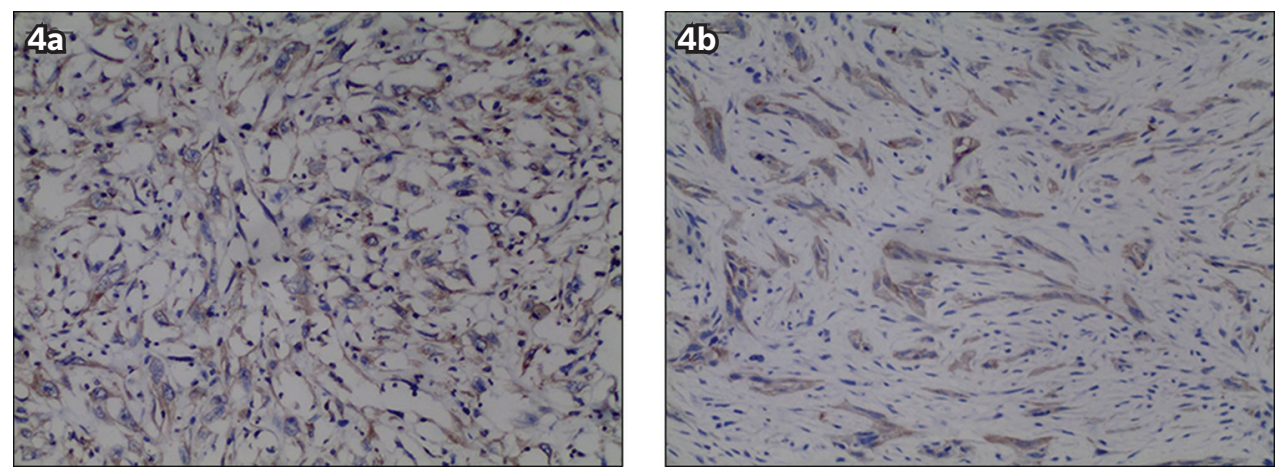

Fig. 4 Photomicrographs of the tumour mass components show (a) immunoreactivity of the sarcomatous areas to vimentin; and (b) cytokeratin expression in the epithelial tumour cells (Immunohistochemical staining, $\times 200$ for both images).

epithelial differentiation, while the spindle stromal element revealed hypercellularity and multinucleated tumour giant cells (Fig. 3). In both, atypical mitoses and pleomorphism were clearly visible. Immunohistochemistry of the malignant stromal elements exhibited abundant expressions of vimentin (VIM), smooth muscle actin (SMA) and S100 protein (Fig. 4a). The epithelial component was positive for cytokeratin (CK) and p63 protein, and staining of the proliferation marker $\mathrm{Ki}-67$ was approximately $60 \%$ of cells
(Fig. 4b). Based on the histological and immunohistochemical findings, the tumour in the left mandible was diagnosed as sarcomatoid carcinoma.

One month later, the patient underwent further radical composite partial mandibulectomy of the left mandibular sarcomatoid carcinoma, and mandibular reconstruction using a bridging plate (Fig. 5). At the same time, cystectomy of the right mandible was performed. Postoperative pathological diagnosis 


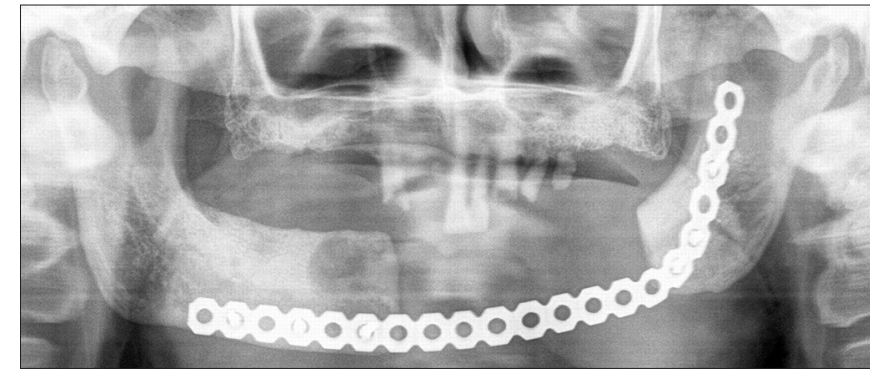

Fig. 5 Panoramic radiograph shows radical composite partial mandibulectomy and mandibular reconstruction with a titanium plate.

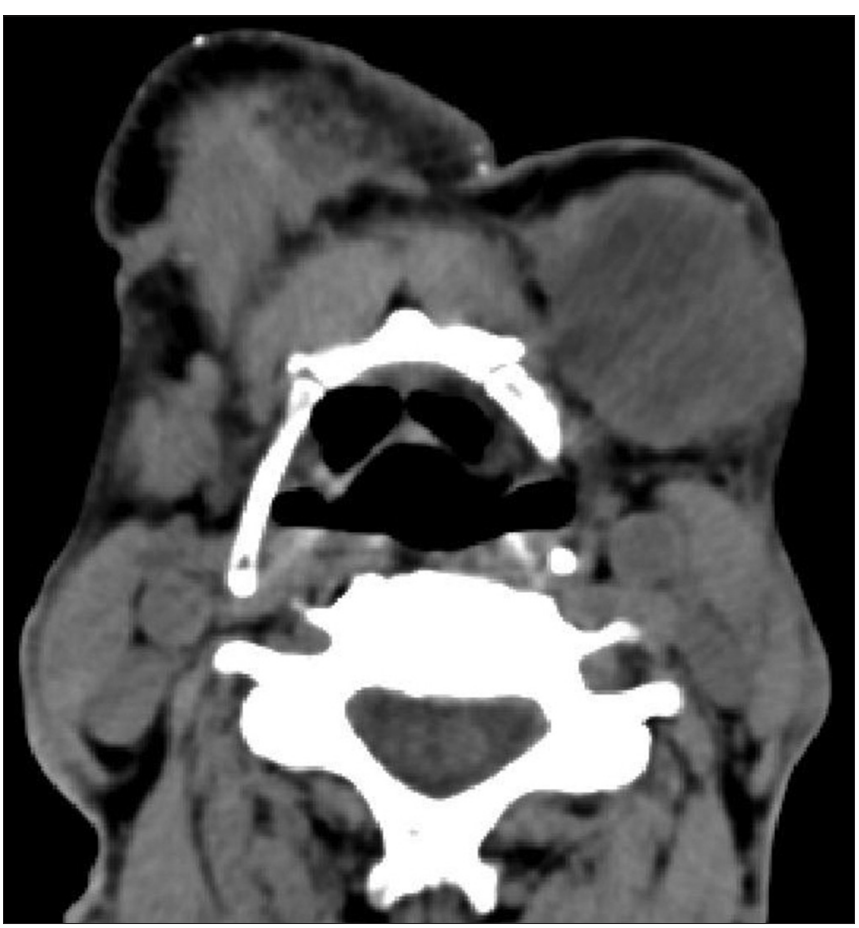

Fig. 6 CT image taken upon re-examination shows tumour recurrence in the submandibular region.

was epithelial odontogenic cyst with local cholesterol cleft reaction.

The patient did not receive subsequent chemotherapy and postoperative irradiation, and died from local recurrence and extensive metastases at eight months post operation (Fig. 6).

\section{DISCUSSION}

Sarcomatoid carcinoma, also termed spindle cell or metaplastic carcinoma, is an uncommon malignant tumour that comprises both malignant epithelial (carcinomatous) and mesenchymal (sarcomatous) components, with or without the presence of heterologous elements. ${ }^{(3-5)}$ The origin of these tumours is controversial. Some studies consider sarcomatoid carcinoma to be merely a collision of sarcoma and carcinoma that have developed independently. Others have suggested that both components arise from an omnipotent cell. ${ }^{(6-8)}$ The 2004 World Health Organization classification of urinary tract tumours does not distinguish between sarcomatoid carcinoma and carcinosarcoma, and uses the term 'sarcomatoid carcinoma' to categorise all of such lesions. ${ }^{(9)}$

The main features of sarcomatoid carcinoma are: (a) the tumour is of epithelial origin; and (b) both the epithelial and mesenchymal cell differentiations exist morphologically. Among a large number of spindle cells, malignant epithelial components such as transitional cell carcinomas, squamous cell carcinomas, adenocarcinomas or anaplastic carcinomas are visible. Ultrastructurally, a transitional zone exists between the carcinomatous and sarcomatous components, the latter generally

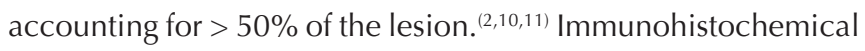
staining shows that the sarcomatous components express epithelial markers, which are important for the diagnosis of sarcomatoid carcinoma. In addition to the expression of the mesenchymal marker VIM, sarcomatous components occasionally express muscle cell or Schwann cell markers such as SMA, actin, neuron-specific enolase and S100 protein, and often express the epithelial marker CK or epithelial membrane antigen. ${ }^{(2,10,12,13)}$

Sarcomatoid carcinoma has a predilection for occurrence in the breast or the urinary, respiratory or digestive tract, and occurrence in the head and neck region is extremely rare. ${ }^{(5,6)}$ To the best of our knowledge, only one case of primary sarcomatoid carcinoma arising from mandibular gingiva has been reported, either as sarcomatoid carcinoma or carcinosarcoma, in the worldwide literature. Stojadinovic et al reported the case of a 67-year-old man whose main tumour mass was located in the gingival mucosa and only focally invaded the bony mandible. Mandibular radiography and CT showed a solid mass and destruction of the left horizontal ramus. The patient was free of tumour recurrence for five years after radical surgery. ${ }^{(3)}$

Our case, the second instance of primary sarcomatoid carcinoma arising from the mandibular gingiva, is similar to Stojadinovic et al's case, in that our patient was also an elderly man and the initial imaging findings were similar to those of the previous case. However, unlike the previous case, the prognosis of our patient was poor - eight months following surgery, our patient died from local tumour recurrence and extensive metastases due to rapid tumour cell growth and diffusion.

Park et al previously reported a case of metastatic mandibular sarcomatoid carcinoma in a 55-year-old man whose panoramic radiograph showed diffuse alveolar bone loss in the left premolar and first molar regions. As his mandibular gingival tumour had metastasised from lung sarcomatoid carcinoma, the patient died of multiple metastatic lesions after three cycles of palliative chemotherapy. ${ }^{(14)}$

In summary, sarcomatoid carcinoma is a biphasic malignant neoplasm with carcinomatous and sarcomatous components. Thus, it has a high degree of malignancy and a poor prognosis, ${ }^{(15)}$ and the risk of local recurrence or distant metastases is also very high. The main radiographic and CT appearance of mandibular sarcomatoid carcinoma is diffuse, irregular osteolytic bone destruction with a soft tissue mass, generally without bone sclerosis or expansion. ${ }^{(3,14)}$ However, these imaging appearances are not unique features of this low-incidence neoplasm. Therefore, the diagnosis of mandibular sarcomatoid carcinoma must depend on histopathological and immunohistochemical examinations. 


\section{REFERENCES}

1. Pelosi G, Sonzogni A, De Pas T, et al. Review article: pulmonary sarcomatoid carcinomas: a practical overview. Int J Surg Pathol 2010; 18:103-20.

2. Thompson LD, Wieneke JA, Miettinen M, Heffner DK. Spindle cell (sarcomatoid) carcinomas of the larynx: a clinicopathologic study of 187 cases. Am J Surg Pathol 2002; 26:153-70.

3. Stojadinovic S, Reinert S, Philippou ST, Machtens E. Carcinosarcoma of the mandibular mucosa: the first report. Int J Oral Maxillofac Surg 2002; 31:444-7.

4. Hansel DE, Epstein JI. Sarcomatoid carcinoma of the prostate: a study of 42 cases. Am J Surg Pathol 2006; 30:1316-21.

5. Noske A, Schwabe M, Pahl S, et al. Report of a metaplastic carcinoma of the breast with multi-directional differentiation: an adenoid cystic carcinoma a spindle cell carcinoma and melanoma. Virchows Arch 2008; 452:575-9.

6. Cheng L, Zhang S, Alexander R, et al. Sarcomatoid carcinoma of the urinary bladder: the final common pathway of urothelial carcinoma dedifferentiation. Am J Surg Pathol 2011; 35:e34-46.

7. Franks TJ, Galvin JR. Sarcomatoid carcinoma of the lung: histologic criteria and common lesions in the differential diagnosis. Arch Pathol Lab Med 2010; 134:49-54.
8. Carter MR, Hornick JL, Lester S, Fletcher CD. Spindle cell (sarcomatoid) carcinoma of the breast: a clinicopathologic and immunohistochemical analysis of 29 cases. Am J Surg Pathol 2006; 30:300-9.

9. World Health Organization Classification of Tumors. Pathology and Genetics of Tumors of the Urinary System and Male Genital Organs. Lyon: IARC Press, 2004

10. Wick MR, Ritter JH, Humphrey PA. Sarcomatoid carcinomas of the lung: a clinicopathologic review. Am J Clin Pathol 1997; 108:40-53.

11. Nagayoshi J, Kawakami T, Maruyama Y. Sarcomatoid carcinoma of the ureter: a case report. Int J Urol 1997; 4:618-20.

12. Wright JL, Black PC, Brown GA, et al. Differences in survival among patients with sarcomatoid carcinoma, carcinosarcoma and urothelial carcinoma of the bladder. J Urol 2007; 178:2302-6.

13. Cornette J, Tjalma WA, Buytaert P. Biphasic sarcomatoid carcinoma or carcinosarcoma of the breast: prognosis and therapy. Eur J Gynaecol Oncol 2005; 26:514-6

14. Park JY, Kim HS, Zo JI, Lee S, Choi SW. Initial presentation of lung sarcomatoid carcinoma as a metastatic lesion in the mandibular gingiva. J Periodontol 2006; 77:734-7.

15. Lee SE, Park SY. Sarcomatoid carcinoma of the small intestine: a rare and highly aggressive tumor. J Korean Surg Soc 2012; 83:321-4. 